\title{
On Steady, Inviscid Shock Waves at Continuously Curved, Convex Surfaces ${ }^{1}$
}

\author{
Barry Koren and Eric van der Maarel \\ Center for Mathematics and Computer Science, \\ P.O. Box 4079, 1009 AB Amsterdam, The Netherlands \\ Communicated by M.Y. Hussaini \\ Received 19 March 1992 and accepted 21 January 1993
}

\begin{abstract}
An accurate and efficient numerical method for steady, two-dimensional Euler equations is applied to study steady shock waves perpendicular to smooth, convex surfaces. The main subject of study is the flow near both ends of the shock wave: the shock-foot and shock-tip flow. A known analytical model of the inviscid shock-foot flow is critically investigated, analytically and numerically. The results obtained agree with those of the existing analytical model. For the inviscid shock-tip flow, two existing analytical solutions are reviewed. Numerical results are presented which agree with one of these two solutions. Good numerical accuracy is achieved through a monotone, second-order accurate, finite-volume discretization. Good computational efficiency is obtained through iterative defect correction iteration and a multigrid acceleration technique which employs local grid refinement.
\end{abstract}

\section{Introduction}

Near shock waves appearing with local supersonic zones in steady, inviscid flows along continuously curved, convex walls (Figure 1), the following two intriguing flow regions exist:

- The flow region where the shock wave abuts the continuously curved, convex wall (the shockfoot region).

- The flow region near the other end of the shock wave (the shock-tip region).

In this section we start by reviewing some known studies of the local flow in both regions. Here we particularly look at aspects such as accuracy and-in the case of numerical studies-also computational efficiency. In the following the shock foot and shock tip are defined as the lower and upper endpoint of the shock wave.

\subsection{Shock-Foot Region}

1.1.1. Normal Shock with Normal Extension. The following physically interesting situation occurs. By assuming that the flow does not change direction when passing through the shock foot, the shock is necessarily normal to the surface and hence, passing through the shock foot, the gas motion satisfies the normal-shock relations. Considering a shock wave which remains normal to some (arbitrary)

\footnotetext{
${ }^{1}$ This work was performed as part of a BRITE/EURAM Area 5 project, under Contract No. AERO-0003/1094.
} 


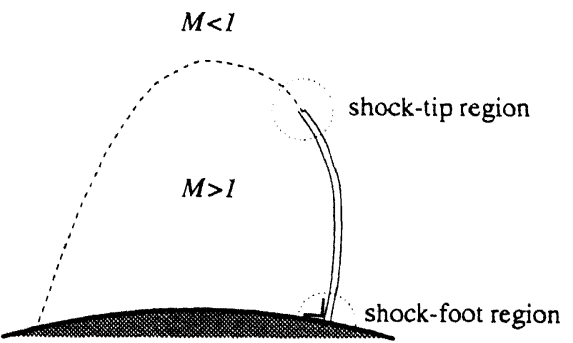

Figure 1. Shock wave fencing off local supersonic zone along continuously curved, convex wall.

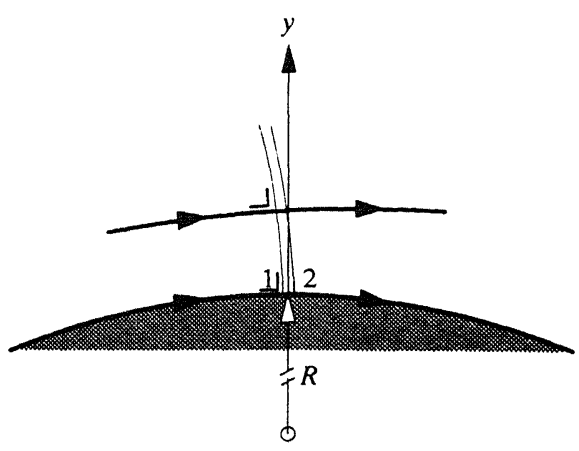

Figure 2. Normal shock with normal extension.

distance above the surface (Figure 2), the normal-shock relations remain valid up to this distance. In the following we refer to such a shock as a normal shock with normal extension.

Along the continuously curved surface sketched in Figure 2, the gas motion also satisfies the equation of curvilinear motion:

$$
\frac{\partial p}{\partial y}=\frac{\gamma p M^{2}}{R}
$$

where $y$ is the coordinate normal to the wall and $R$ is the radius of curvature of the wall. (Throughout this paper, $R$ is assumed to be finite.)

It may be apparent that for a normal shock with normal extension, the postshock flow which has just satisfied the normal-shock relations, does not necessarily satisfy (1) any longer. We show that this is indeed the case by deriving that, for a normal shock wave with normal extension, only one specific Mach number at the upstream shock-foot face exists, for which passage through the normal-shock foot does not lead to a conflict with respect to satisfaction of the equation of curvilinear motion in the downstream shock-foot face. We introduce the subscripts 1 and 2 for the upstream and downstream shock-foot face, respectively (Figure 2). Applying (1) at the downstream shock-foot face, we have

$$
\frac{\partial p_{2}}{\partial y}=\frac{\gamma p_{2} M_{2}^{2}}{R} .
$$

Alternatively, we can also write, for $\partial p_{2} / \partial y$,

$$
\frac{\partial p_{2}}{\partial y}=\frac{\partial}{\partial y}\left(\frac{p_{2}}{p_{1}}\right) p_{1}+\frac{p_{2}}{p_{1}} \frac{\partial p_{1}}{\partial y} .
$$

We proceed by expressing $\partial p_{2} / \partial y$ according to (2a) and (2b) in terms of the preshock flow. For the postshock terms $p_{2}$ and $M_{2}^{2}$ occurring in (2a) and $(2 \mathrm{~b})$, we can directly write the normal-shock relations:

$$
\begin{aligned}
p_{2} & =\frac{2 \gamma M_{1}^{2}-(\gamma-1)}{\gamma+1} p_{1}, \\
M_{2}^{2} & =\frac{2+(\gamma-1) M_{1}^{2}}{2 \gamma M_{1}^{2}-(\gamma-1)} .
\end{aligned}
$$

Further, for the pressure gradient $\partial p_{1} / \partial y$ in $(2 \mathrm{~b})$, we have

$$
\frac{\partial p_{1}}{\partial y}=\frac{\gamma p_{1} M_{1}^{2}}{R} .
$$

The gradient term $(\partial / \partial y)\left(p_{2} / p_{1}\right)$ in $(2 \mathrm{~b})$ requires some work. Given the normal extension of the shock wave, we may use the normal-shock relation (3a) for rewriting this gradient term. It follows that $(\partial / \partial y)\left(p_{2} / p_{1}\right)=(4 \gamma /(\gamma+1)) M_{1}\left(\partial M_{1} / \partial y\right)$, which-assuming the preshock flow to be homentropic and 
applying (3c) -leads to

$$
\frac{\partial}{\partial y}\left(\frac{p_{2}}{p_{1}}\right)=\frac{-4 \gamma}{\gamma+1}\left(1+\frac{\gamma-1}{2} M_{1}^{2}\right) \frac{M_{1}^{2}}{R}
$$

Equating (2a) and (2b) and substituting (3a)-(3d) yields the following compact quadratic equation for $M_{1}^{2}$ :

$$
M_{1}^{4}-(\gamma+1) M_{1}^{2}-1=0 .
$$

From (4) it follows that the only Mach number $M_{1}$ - to be further denoted by $M_{1}^{*}$-which allows a normal shock wave with normal extension, is

$$
M_{1}^{*}=\sqrt{\frac{\gamma+1}{2}+\sqrt{\left(\frac{\gamma+1}{2}\right)^{2}+1}} .
$$

In more cumbersome derivations, this specific Mach number was already found by Tsien (1947), Lin and Rubinov (1948), and Zierep (1958a).

1.1.2. Normal Shock with Oblique Extension. Concerning the flow in the shock-foot region for Mach numbers $M_{1}$ different from $M_{1}^{*}$, Zierep (1958a) obtained further analytical results. In this section we review some of these results.

Zierep (1958a) found the known result that for $M_{1} \neq M_{1}^{*}$-in general一in its foot the shock wave has an infinitely large curvature. In analogy to the normal shock with normal extension as depicted in Figure 2, the present shock can be illustrated as in Figure 3. At an arbitrarily small distance above the surface it already has a finite degree of obliquity. This singular change from normal shock wave (in the foot) to oblique shock wave (immediately above the foot) implies that at the downstream shock-foot face an infinitely steep decrease in the surface pressure occurs, which ensures that the conflict that was mentioned in the previous section does not occur. In the following we refer to shocks of this type as normal shocks with oblique extension.

Support for the correctness of this singular solution is sometimes believed to come from the fact that postshock expansions (though not infinitely steep, as in Figure 4(a)) are really observed in the results of, e.g., wind-tunnel experiments. (Zierep (1958a) explicitly makes a reference to the classical experimental results of Ackeret et al. (1946).) In our opinion the physics in wind-tunnel experiments is too different from inviscid physics to be of much support for the correctness of an inviscid analysis. Instead of from, e.g., wind-tunnel experiments, support for at least the singular behavior of the postshock surface pressure simply comes from theory. Namely, if the postshock pressure correction has a finite steepness (Figure 4(b)), there would have to be a finite region in which the equation of curvilinear motion is not satisfied, which-in a steady flow-is physically impossible.

From a theoretical point of view some questions still exist with respect to the specific singular solution found by Zierep. First, although Zierep's analysis starts with the full Euler equations, in becoming local (i.e., in approaching the wall along the shock wave), it applies to these starting

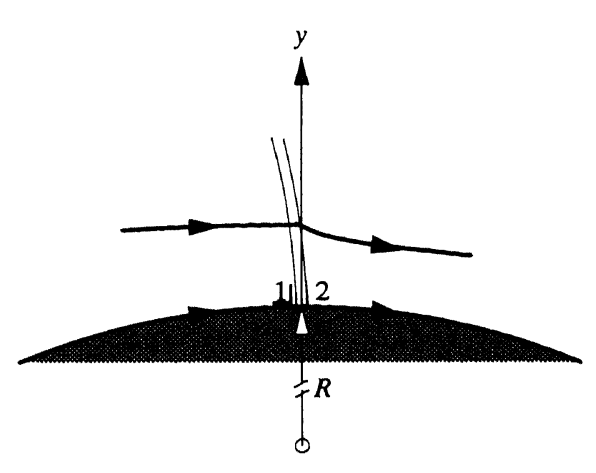

Figure 3. Normal shock with oblique extension.
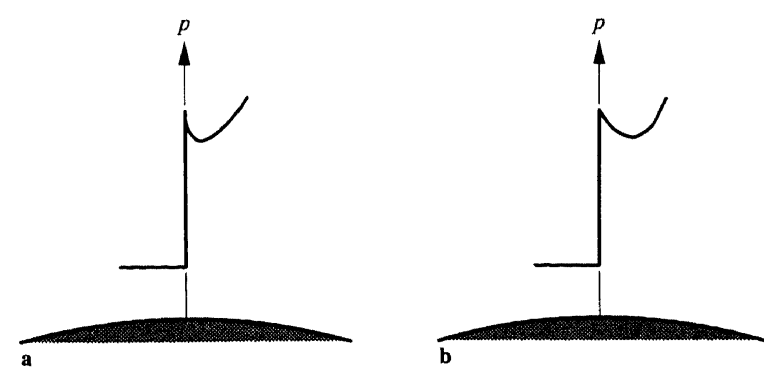

Figure 4. Postshock surface pressure corrections: (a) infinitely steep expansion and (b) finitely steep expansion. 


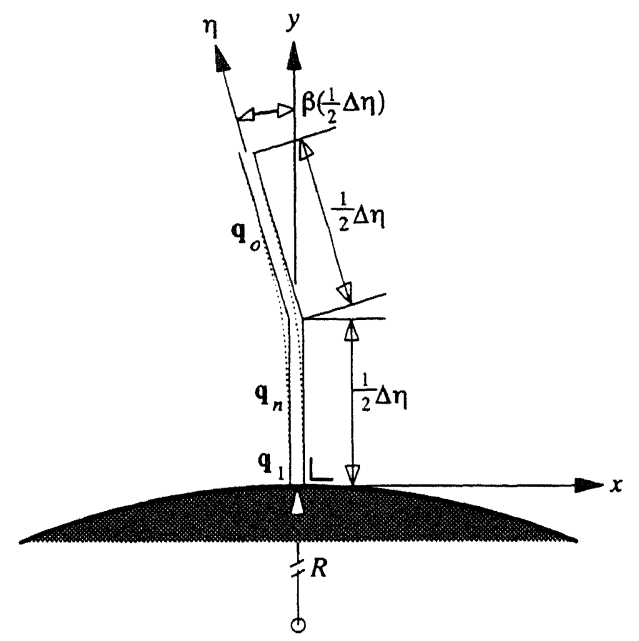

Figure 5. Bisegmental piece of shock wave.

equations some simplifications which are based on questionable preassumptions about how the flow locally behaves there. Second, according to Zierep's solution, the normal shock with oblique extension cannot exist in the range $\left(M_{1}^{*}, M_{1}^{* *}\right)$, with $M_{1}^{*}$ satisfying relation (5) and with $M_{1}^{* *}$ satisfying

$$
M_{1}^{* *}=\sqrt{\frac{2+(\gamma+1) / 2}{2-(\gamma+1) / 2}} .
$$

(Notice that for any admissible value of $\gamma$-i.e., any $\gamma$ from the range $[1,3]$-it holds that $M_{1}^{*}<$ $M_{1}^{* *}$.) Though we do not know any example of a steady shock wave normal on a convex surface in the $M_{1}$-range $\left(M_{1}^{*}, M_{1}^{* *}\right)$, a physical understanding of this remarkable range of Mach numbers is still missing. Further, concerning the upper bound $M_{1}^{* *}$ of this range, in the limit $M_{1} \downarrow M_{1}^{* *}$ Zierep's solution becomes so much singular that our conjecture is that the corresponding solution conflicts with the standard, uncurved-shock relations that were used in deriving it. To give some evidence of this probable discrepancy we consider the bisegmental piece of shock wave depicted in Figure 5. This stylized piece of shock wave is to be regarded as a discretization of the exact shock wave, indicated by dotted lines in Figure 5. The bisegmental piece of shock wave consists of a segment which stands normal on the wall and with an oblique segment on top of that. The foot of the normal segment and the tip of the oblique segment are tangent to the exact shock wave. The segments have the same length, so that the kink in the discrete shock wave is centered. For this discrete, composite shock we derive an expression for the transport of mass, $x$-momentum, and $y$-momentum across its upstream face, per unit of length and time. (Concerning the transport of energy, the flow is assumed to be isenthalpic.) For this derivation, along the exact shock wave we introduce the coordinate $\eta$, with the shock foot as origin. Next, we introduce the average gas states

$$
\begin{aligned}
& \mathbf{q}_{\mathrm{n}} \equiv \frac{\int_{0}^{\Delta n / 2} \mathbf{q}(\eta) d \eta}{\frac{1}{2} \Delta \eta}, \\
& \mathbf{q}_{\mathrm{o}} \equiv \frac{\int_{\Delta \eta / 2}^{\Delta \eta} \mathbf{q}(\eta) d \eta}{\frac{1}{2} \Delta \eta}
\end{aligned}
$$

at the upstream face of the normal and oblique segment, respectively (Figure 5). Then, for this transport vector per unit of length and time, to be further denoted by $\mathbf{F}$, we can write

in which

$$
\mathbf{F}=\frac{1}{2} \mathbf{f}\left(\mathbf{q}_{\mathrm{n}}\right)+\frac{1}{2} \cos \beta \mathbf{f}\left(\mathbf{q}_{\mathrm{o}}\right)+\frac{1}{2} \sin \beta \mathbf{g}\left(\mathbf{q}_{\mathrm{o}}\right)
$$

$$
\begin{aligned}
\mathbf{f}(\mathbf{q}) & =\left(\rho u, \rho u^{2}+p, \rho u v\right)^{T} \\
\mathbf{g}(\mathbf{q}) & =\left(\rho v, \rho v u, \rho v^{2}+p\right)^{T}
\end{aligned}
$$

with $u$ and $v$ being the velocity components in the $x$ - and $y$-directions, respectively (Figure 5). In (8) 
the last two terms are due to the curvature of the shock wave. For the angle $\beta$ in these two curvature terms we use the detailed expression $\beta=\beta(\eta)$ given in Zierep (1958b) and we study the behavior of $F$ in the limit $\Delta \eta \downarrow 0$. For $\beta=\beta(\eta)$, Zierep (1958b) gives

$$
\beta(\eta)=\sqrt{2 \sigma \frac{\eta}{R}}
$$

where, rewriting from Zierep (1958b),

$$
\sigma=\sigma\left(M_{1}\right)=\frac{\lambda\left(M_{1}\right)-\frac{(\gamma+1)\left(M_{1}^{2}-1\right)\left(\gamma+\left(\left(\gamma^{2}+1\right) /(\gamma+1)\right)\left(M_{1}^{2}-1\right)\right)}{1-\gamma+(1+3 \gamma) M_{1}^{2}}}{1-2 \frac{\left(M_{1}^{2}-1\right)\left(\gamma+\left(\left(\gamma^{2}+1\right) /(\gamma+1)\right)\left(M_{1}^{2}-1\right)\right)}{\left(1-\gamma+(1+3 \gamma) M_{1}^{2}\right)\left(1+((\gamma-1) /(\gamma+1))\left(M_{1}^{2}-1\right)\right)}},
$$

with

$$
\lambda\left(M_{1}\right)=\frac{\left(2+(\gamma-1) M_{1}^{2}\right)\left(1+\gamma M_{1}^{2}\right)}{1-\gamma+(1+3 \gamma) M_{1}^{2}} .
$$

As an example, in Figure 6 we depict $\sigma\left(M_{1}\right)$ for a di-atomic gas $\left(\gamma=\frac{7}{5}\right)$, for which (5) and (6) yield $M_{1}^{*} \approx 1.662, M_{1}^{* *}=2$. Given (11a) and (11b), we verify:

- That a normal shock wave with normal extension, as considered in Section 1.1.1, can only occur for $M_{1}^{*}$, because $\sigma\left(M_{1}\right)=0$ for $M_{1}=M_{1}^{*}$ only.

- That no steady shock wave perpendicular to the wall can occur in the $M_{1}$-range $\left(M_{1}^{*}, M_{1}^{* *}\right)$ (the shaded range in Figure 6) because $\sigma\left(M_{1}\right)<0$ holds there.

- That a very singular shock-foot flow occurs in the limit $M_{1} \downarrow M_{1}^{* *}$, since $\lim _{M_{1} \downarrow M_{1}^{* *}} \sigma\left(M_{1}\right)=\infty$.

- That the shock wave has infinite curvature in its foot for all (supersonic) $M_{1}<M_{1}^{*}$ and all $M_{1}>M_{1}^{* *}$ because $\lim _{\eta \downarrow 0}(d \beta / d \eta)=\sqrt{\sigma\left(M_{1}\right) /(2 \eta R)}=\infty$ for all these values of $M_{1}$.

Zierep (1958a) concludes that the curvature of the shock wave in its foot at $M_{1}=M_{1}^{*}$ is zero. In our opinion, this conclusion cannot be drawn from Zierep's theory, because in $\lim _{M_{1} \uparrow M_{1}^{*}} \lim _{\eta \downarrow 0}(d \beta / d \eta)=$ $\sqrt{\sigma\left(M_{1}\right) /(2 \eta R)}=0 / 0$ both limits can be taken strictly independent from each other $\left(M_{1}\right.$ does not depend on $\eta$ ) and hence $\lim _{M_{1} \uparrow M_{1}^{*}} \lim _{\eta \downarrow 0}(d \beta / d \eta)$ is undetermined. For a finite (or even zero) shock curvature in the shock foot, $\sigma=0$ is a necessary but insufficient condition.

With (10) and the Taylor-series expansion

$$
\mathbf{q}(\eta)=\mathbf{q}_{1}+\eta \frac{\partial \mathbf{q}_{1}}{\partial \eta}+\mathbf{O}\left(\eta^{2}\right),
$$

where $\mathbf{q}_{1}$ is the gas state at the upstream shock-foot face (Figure 5), through successively (7a), (7b) and

Figure 6. $\sigma=\sigma\left(M_{1}\right)$ from Zierep (1958b), for $\gamma=\frac{7}{5}$.

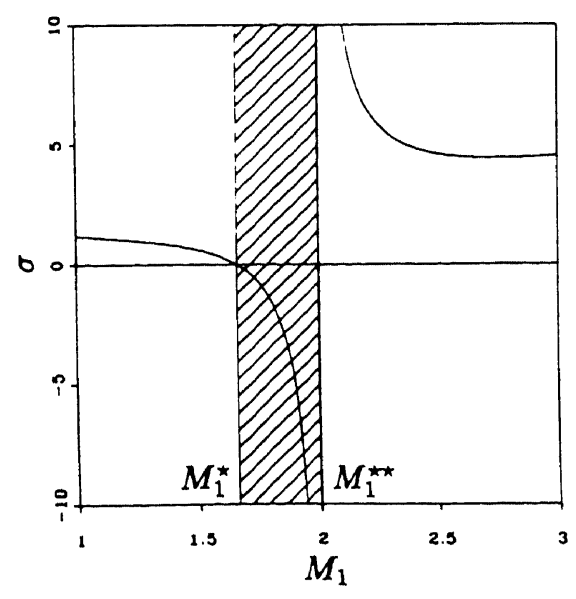


(9a), (9b), relation (8) for the flux vector $\mathbf{F}$ can be written as

$$
\begin{aligned}
\mathbf{F}=\left[\mathbf{f}\left(\mathbf{q}_{1}\right)+\frac{1}{2} \Delta \eta \frac{\partial \mathbf{f}\left(\mathbf{q}_{1}\right)}{\partial \eta}+\mathbf{O}\left(\Delta \eta^{2}\right)\right] & +\frac{1}{2}\left[\cos \left(\sqrt{\sigma \frac{\Delta \eta}{R}}\right)-1\right]\left[\mathbf{f}\left(\mathbf{q}_{1}\right)+\frac{3}{4} \Delta \eta \frac{\partial \mathbf{f}\left(\mathbf{q}_{1}\right)}{\partial \eta}+\mathbf{O}\left(\Delta \eta^{2}\right)\right] \\
& +\frac{1}{2} \sin \left(\sqrt{\sigma \frac{\Delta \eta}{R}}\right)\left[\mathbf{g}\left(\mathbf{q}_{1}\right)+\frac{3}{4} \Delta \eta \frac{\partial \mathbf{g}\left(\mathbf{q}_{1}\right)}{\partial \eta}+\mathbf{O}\left(\Delta \eta^{2}\right)\right] .
\end{aligned}
$$

From (13) it appears that, for $\sigma<\infty$, in the limit $\Delta \eta \downarrow 0$-despite the infinite curvature of the shock - the standard, uncurved-shock relations can still be applied; the curvature terms vanish:

$$
\begin{aligned}
\lim _{\Delta \eta \downarrow 0} \mathbf{F}_{\sigma<x}= & \lim _{\Delta \eta \downarrow 0}\left[\mathbf{f}\left(\mathbf{q}_{1}\right)+\frac{1}{2} \Delta \eta \frac{\partial \mathbf{f}\left(\mathbf{q}_{1}\right)}{\partial \eta}+\mathbf{O}\left(\Delta \eta^{2}\right)\right] \\
& +\lim _{\Delta \eta \downarrow 0}\left[-\frac{1}{4} \sigma \frac{\Delta \eta}{R}+\mathbf{O}\left(\Delta \eta^{2}\right)\right]\left[\mathbf{f}\left(\mathbf{q}_{1}\right)+\frac{3}{4} \Delta \eta \frac{\partial \mathbf{f}\left(\mathbf{q}_{1}\right)}{\partial \eta}+\mathbf{O}\left(\Delta \eta^{2}\right)\right] \\
& +\lim _{\Delta \eta \downarrow 0}\left[\sqrt{\sigma \frac{\Delta \eta}{R}}+\mathbf{O}\left(\Delta \eta^{3 / 2}\right)\right]\left[\mathbf{g}\left(\mathbf{q}_{1}\right)+\frac{3}{4} \Delta \eta \frac{\partial \mathbf{g}\left(\mathbf{q}_{1}\right)}{\partial \eta}+\mathbf{O}\left(\Delta \eta^{2}\right)\right]=\mathbf{f}\left(\mathbf{q}_{1}\right) .
\end{aligned}
$$

However, from (13) it also appears that, for $\sigma=\infty$, in the limit $\Delta \eta \downarrow 0$ the influence of shock curvature cannot be neglected. This shows the discrepancy of the Zierep model in the limit $M_{1} \downarrow M_{1}^{* *}$.

1.1.3. Numerical Shock-Foot Study. In our numerical computations we apply the full, steady twodimensional Euler equations in the complete domain. In doing so, we look at the existence of a postshock expansion as a function of $M_{1}$ and-if existing-its steepness for decreasing mesh width. To get a good impression of the expansion's steepness for decreasing mesh width, without making excessive computational costs, we apply a technique which allows us to zoom in at local flow features efficiently: a solution-adaptive multigrid technique. A description of this technique is given in Section 2.

\subsection{Shock-Tip Region}

Concerning the shock-tip region, today some uncertainties still exist about how the shock wave merges into the main flow. Some authors claim that the shock wave becomes weaker in the direction of the sonic line (Figure 7(a)). Others claim that it bifurcates such that a triple-shock configuration (upside-down $\lambda$-configuration) occurs (Figure 7(b)). Notice that a triple-shock configuration must contain at least one additional discontinuity. In Courant and Friedrichs (1976, pp. 332-333) it is proved that three zones of different, continuous steady states are impossible. (In Figure 7(b) we added a contact discontinuity.)

Some profound analytical studies exist which support either the configuration in Figure 7(a) or that in Figure 7(b). Representative examples of these studies are those of Germain and Gillon (1961) and Kraiko (1985), respectively. Germain and Gillon (1961) consider the transonic small perturbation (TSP) equation and find, through a hodograph method, that the connection of the sonic line to the shock wave is, in general, tangent and such that the shock tip is a point of inflection (i.e., at their connection, shock wave and sonic line have oppositely signed curvatures). According to Germain and
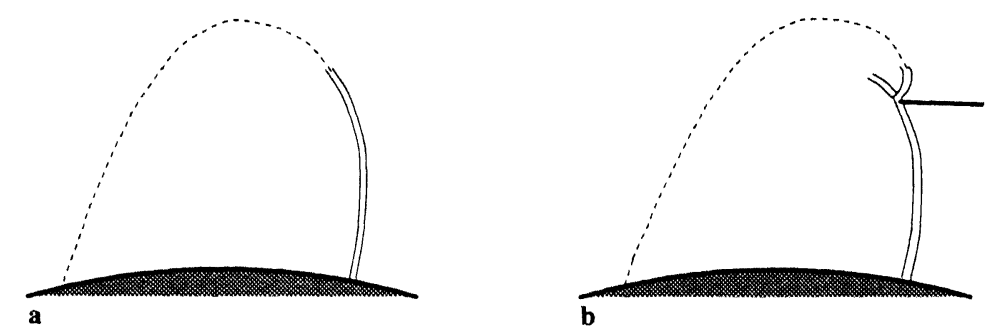

Figure 7. Two types of shock-tip configurations: (a) single-shock and (b) triple-shock. 
Gillon (1961), the entire shock wave is of a strong type (therefore with transition from supersonic to subsonic speeds only). They conclude that other types of shock-tip configurations are possible, but only in exceptional cases. Opposed to this, Kraiko (1985) finds that the triple-shock-tip configuration sketched in Figure 7(b) is the commonly occurring one. Most present-day studies are in favor of this $\lambda$-configuration being the most common one. A superficial physical reasoning supporting this is the known fact that, in case of a convex surface, the compression waves (the waves propagating from the sonic line) are known to taper faster than the expansion waves (the waves running from the surface). The single-shock configuration in Figure 7(a) may be regarded as a special case of the triple-shock configuration, namely, with the shock-triple-point in the highest possible position.

Of all the analytical studies on shock-tip configurations known to us, the shortcomings are their localness and use of rather simplified equations, such as the TSP equation. Investigations of the local shock-tip flow as an integral part of a much more global flow have already been made numerically, though still using the TSP equation. Examples of such numerical studies are those of Murman (1974), Hafez and Cheng (1975), and Yu and Seebass (1976). Whereas the local tip flow certainly can be described by good approximation as a potential flow, the global flow generally cannot. It makes sense to consider the full Euler equations not only for studying the shock-foot flow, but also for studying the shock-tip flow. In the numerical shock-tip work known to us, as well as no use being made of the Euler equations, no use is made of locally refined grids also. As a consequence, all of these numerical results are also limited in accuracy by the use of relatively coarse grids. It makes sense to apply a local grid refinement technique, not only for the shock-foot flow, but also for the shock-tip flow.

\section{Solution-Adaptive Multigrid Technique}

\subsection{Review}

The following two types of solution-adaptive grid methods exist: (i) grid movement methods and (ii) grid enrichment methods. The difference between both methods is that grid movement methods try to get a maximal accuracy for a fixed cost (a fixed number of grid points), whereas grid enrichment methods try to attain a fixed accuracy for a minimal cost (a minimal number of grid points). Difficulties of grid movement methods are: the simultaneous accurate resolution of more than one flow feature, and the control over grid properties which are of importance to the specific discretization method considered (grid properties such as, e.g., smoothness and orthogonality). Difficulties of grid enrichment techniques are: to keep track (in the data structure) of the local refinements, and to assure accuracy and conservation at the fine-coarse grid boundaries.

For the present flow features of interest (shock waves) we prefer to apply grid enrichment. Concerning grid enrichment, two types of methods can also be considered: (i) nested methods and (ii) unnested methods. Both methods apply fine subgrids overlying coarser grids. Whereas in the nested approach these fine grids are by definition aligned with the underlying coarser grids, in the unnested approach they are not necessarily so. An example of an unnested method in which (multidimensional) fluid flow equations are considered is the method of Fuchs (1990). Examples of nested methods considering (multidimensional) fluid flow equations are the methods of Berger and Jameson (1985), De Zeeuw and Powell (1991), Warren (1992), and Van der Maarel (1992). Nested enrichment has as an advantage over the unnested approach, its direct allowance for standard geometric multigrid acceleration. (This possibility is exploited in the methods of Warren (1992) and Van der Maarel (1992).) Besides its use for acceleration, another interesting possibility of the multigrid in this context is to apply it for estimating the local truncation error to be used for the refinement criterion in smooth flow regions. Further, the nested approach also has the advantage that satisfaction of accuracy and conservation requirements across aligned fine-coarse grid boundaries is more easily achieved than across nonaligned boundaries. For the present application we prefer nested enrichment; we proceed by discussing it further.

Without special care, in general, the set of nested, locally refined cells will be unstructured. Yet, structure can be obtained by properly clustering cells which are flagged to be refined. (For details on clustering, see Berger and Oliger, 1984.) The previously mentioned method of Berger and Jameson 
(1985) is an example of an embedded mesh method; a method which applies clustering. The methods of De Zeeuw and Powell (1991), Warren (1992), and Van der Maarel (1992) are examples of cellular refinement methods; methods that do not apply clustering. Concerning the efficiency of cellular and embedded mesh refinement methods, it is not clear to us which is most efficient. An obvious efficiency drawback of the cellular refinement approach is that it is necessary to keep track of all refined cells (as opposed to all refined subgrids only). Against that, clustering has of course the drawback of refining cells which are not actually flagged to be refined. Besides that, clustering may already, by its use only, significantly increase the computational overhead. A major efficiency advantage of embedded mesh refinement is then often said to come from its better possibilities for supercomputing. For vectorization, this is certainly true. For parallelization-in our opinion-the possibilities are the same. Concerning vectorization of a cellular refinement code, the gain in computing time is not necessarily nil. Recently, some work has been done by Lioen and Louter-Nool (to appear) on vectorization of the code applied in Van der Maarel (1992). It was found that automatic vectorization of this code does not give much gain. However, by developing some sort of gather software, on a CRAY-YMP, Lioen and Louter-Nool still obtained a speed-up of approximately a factor of four.

Because of its satisfactory application to another singular flow problem (Van der Maarel and Koren, 1991), in the present paper we adhere to the cellular refinement method of Van der Maarel.

\subsection{Data Structure}

A detailed description of the data structure of the nested enrichment method of Van der Maarel (1992) is given in Hemker et al. (1990). Here a summary is given. Consider the steady, twodimensional Euler equations on a domain $\Omega \subset \mathbb{R}^{2}$ with boundary $\partial \Omega$. In the discretization, $\Omega$ is approximated by a regular partition of disjoint quadrilateral cells. Grids on different levels of refinement are considered, each level employing a regular partition of one or more subdomains of $\Omega$. The grid on level $l$ is denoted by $\Omega_{l}, l \in\{0,1,2, \ldots, \Lambda, \ldots, L\}$. The grids $\Omega_{0}$ up to and including $\Omega_{\Lambda}$ completely cover $\Omega$. $\Omega_{0}$ is the coarsest grid and $\Omega_{\Lambda}$ is called the basic grid. Starting from $\Omega_{\Lambda+1}$ up to and including the finest grid $\Omega_{L}$, the grids do not necessarily cover the complete domain $\Omega$.

For $l>0$, each cell of $\Omega_{l}$ is a member of a disjoint division (into a set of $2 \times 2$ smaller cells) of a cell of $\Omega_{l-1}$. The cells of $\Omega_{l-1}$ and $\Omega_{l}$ are coexistent, i.e., coarse cells are not removed from the system when they are overlaid by fine cells. All cells in the geometric structure are related to each other through a so-called quad-tree data structure. The location of each cell of $\Omega_{l}$ is determined by a set of coordinates $(i, j) \in \mathbb{Z}^{2}$. The refinements of cell $\left(\Omega_{l}\right)_{i, j}$ are $\left(\Omega_{l+1}\right)_{2 i, 2 j},\left(\Omega_{l+1}\right)_{2 i+1,2 j},\left(\Omega_{l+1}\right)_{2 i, 2 j+1}$, and $\left(\Omega_{l+1}\right)_{2 i+1,2 j+1}$. The edge $\left(\partial \Omega_{l}\right)_{i, j}$ of $\left(\Omega_{l}\right)_{i, j}$ consists of four rectilinear sides. The grid $\Omega_{l}$ consists of a part $\Omega_{l}^{\mathrm{f}}$ with cells that have been refined, and a part $\Omega_{l}^{\mathrm{c}}$ with cells that have not been refined. The grids $\Omega_{l}^{\mathrm{f}}$ and $\Omega_{l+1}$ cover the same part of $\Omega$. The set of all nonrefined cells is called the composite grid. It is the solution on the composite grid that we want to compute.

\subsection{Discretization Method and Solution Method}

2.3.1. Earlier, Nonadaptive Method. The discretization method and solution method applied in Van der Maarel (1992) are based on methods presented in the earlier nonadaptive work of Hemker and Spekreijse (1986) and Koren (1988). In this earlier work a cell-centered finite-volume discretization is used, in combination with an upwind numerical flux function. The solution method applied requires that the flux function is continuously differentiable. The scheme of Osher and Solomon (1982) satisfies this requirement. At each cell face this scheme approximately solves a one-dimensional Riemann problem. The discretization is first-order accurate by taking the left and right Riemann states equal to those in the corresponding adjacent finite volumes. Second-order accuracy is obtained by applying second-order accurate, piecewise polynomial state interpolation. To preserve monotonicity of the solutions, a limiter is used. We proceed by describing the nonadaptive discretization in more detail for the scalar, linear, one-dimensional model equation

$$
\frac{\partial u}{\partial x}=0
$$


(A one-dimensional description suffices because in going to multidimensionality the directionally split one-dimensional upwind approach is followed.) On an equidistant, cell-centered finite-volume grid $\Omega_{l}=\bigcup_{i=1}^{n} \Omega_{i}$, the discrete equation corresponding to (15) reads

$$
u_{i+1 / 2}-u_{i-1 / 2}=0
$$

where the half-integer indices refer to the cell faces. For the cell face flux $u_{i+1 / 2}$, the limited, second-order accurate discretization takes

$$
u_{i+1 / 2}=u_{i}+\frac{1}{2} \psi\left(R_{i+1 / 2}\right)\left(u_{i}-u_{i-1}\right), \quad R_{i+1 / 2}=\frac{u_{i+1}-u_{i}}{u_{i}-u_{i-1}} .
$$

For the limiter $\psi(R)$ the discretization takes that of Van Albada et al. (1982):

$$
\psi(R)=\frac{R^{2}+R}{R^{2}+1} .
$$

Scheme (17a)-(17b) is called the Van Albada scheme. The Van Albada scheme belongs to the class of limited $\kappa=0$ schemes, which are known to give optimal convergence of iterative defect correction (IDeC) (see Désidéri and Hemker, 1990). Notice that scheme (17a)-(17b) cannot be applied at the near-inlet cell face $\partial \Omega_{3 / 2}$ and at the outlet cell face $\partial \Omega_{n+1 / 2}$, since $\Omega_{0}$ and $\Omega_{n+1}$ do not exist. (At the inlet cell face, $\partial \Omega_{1 / 2}$, we assume the solution to be known from the inlet boundary condition.) At $\partial \Omega_{3 / 2}$ and $\partial \Omega_{n+1 / 2}$, it is taken that

$$
\begin{aligned}
u_{3 / 2} & =\frac{1}{2}\left(u_{1}+u_{2}\right), \\
u_{n+1 / 2} & =u_{n}+\frac{1}{2}\left(u_{n}-u_{n-1}\right) .
\end{aligned}
$$

With this, formally, a local reduction to first-order accuracy occurs in $\Omega_{1}, \Omega_{2}$, and $\Omega_{n}$. Fortunately, from numerical experiments for the Euler equations, it appears that this order reduction does not do any harm. (To give some evidence of this, we refer to the surface entropy and surface pressure distributions as presented in Koren (1988) for the standard NACA0012-airfoil flow at $M_{\infty}=0.8$, $\alpha=1.25^{\circ}$.)

The multigrid method which is applied for solving the system of discretized equations on globally refined grids is nonlinear multigrid (NMG), with collective symmetric point Gauss-Seidel relaxation as the smoother. This method is very efficient for the first-order discretized Euler equations. Difficulties arising when applying it to the second-order discretized equations are circumvented by introducing IDeC as an outer iteration to NMG. The solution process is started on the coarsest grid and continued (through nested iteration) to the finest grid. In the nested iteration the equations solved are first-order accurate only. After having solved the first-order discretized equations on the finest grid, the second-order discretized equations are solved (through IDeC). Per IDeC cycle only one NMG cycle is applied. (In Koren (1988) this IDeC cycling was found to be the most efficient.)

2.3.2. Present, Adaptive Method. In the present solution-adaptive multigrid method, an important new numerical ingredient is the computation of the left and right cell face states at the fine-coarse grid boundaries. In Van der Maarel (1993) a detailed description and accuracy analysis is given of the treatment of these inner boundaries. Here we summarize its main features. A difference of only one grid level is accepted across a fine-coarse grid boundary. Along these boundaries, by proper interpolation from the local coarse-grid cell-center states, the locally missing fine-grid cell-center states are computed. To maintain global second-order solution accuracy, this interpolation needs to be third-order accurate at least. Considering the fine-coarse grid boundary $\left(\partial \Omega_{l+1}\right)_{2 i+3 / 2,2 j+1}$ (Figure 8), in order to apply the Van Albada scheme the states in the two virtual cells $\left(\Omega_{l+1}\right)_{2 i+2,2 j+1}$ and $\left(\Omega_{l+1}\right)_{2 i+3,2 j+1}$, say $\left(u_{l+1}\right)_{2 i+2,2 j+1}$ and $\left(u_{l+1}\right)_{2 i+3,2 j+1}$, need to be computed. The virtual state $\left(u_{l+1}\right)_{2 i+2,2 j+1}$ is computed through the third-order accurate interpolation formula

$$
\left(u_{l+1}\right)_{2 i+2,2 j+1}=\frac{1}{16}\left[\left(u_{l}\right)_{i, j}+17\left(u_{l}\right)_{i+1, j}-2\left(u_{l}\right)_{i+2, j}+\left(u_{l}\right)_{i, j+1}+\left(u_{l}\right)_{i+1, j+1}-2\left(u_{l}\right)_{i+1, j-1}\right],
$$

and a similar formula is used for $\left(u_{l+1}\right)_{2 i+3,2 j+1}$. 


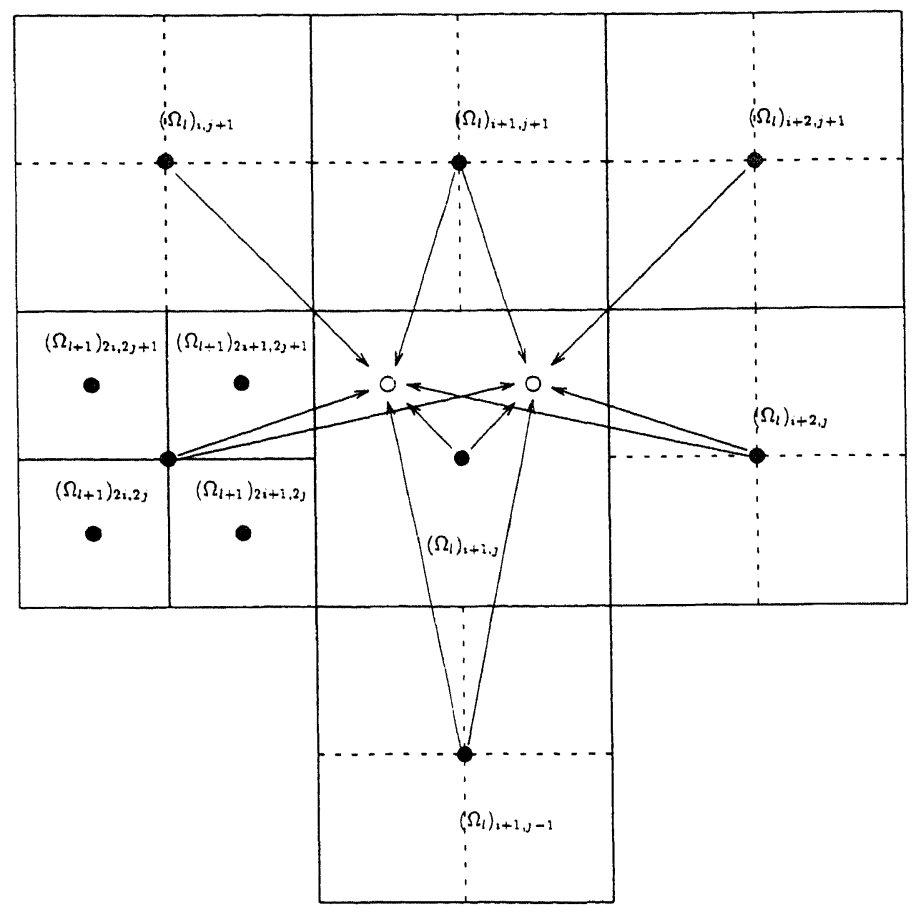

Figure 8. Third-order accurate virtual state interpolation in virtual cells $\left(\Omega_{l+1}\right)_{2 i+2,2 j+1}$ and $\left(\Omega_{l+1}\right)_{2 i+3,2 j+1}$.

To solve the system of discrete equations on locally refined grids, an identical method to that used on globally refined grids is used: IDeC with one NMG cycle per IDeC cycle, and NMG with point Gauss-Seidel relaxation as the smoother. Also here, the solution process is started on the coarsest grid $\Omega_{0}$. It is continued (through nested iteration) to the basic grid $\Omega_{\Lambda}$. In the nested iteration the equations solved are also first-order accurate only. After having solved the first-order discretized equations on $\Omega_{\Lambda}$, the second-order discretized equations are solved (through IDeC), and-upon convergence - based on some appropriate refinement criterion, the locally refined grid $\Omega_{\Lambda+1}$ is introduced, after which IDeC is converged again. Upon convergence of both $\Omega_{\Lambda+1}$ and IDeC, based on the refinement criterion, the locally refined grid $\Omega_{\Lambda+2}$ is introduced, after which IDeC is converged again. Next, upon (eventually new) convergence of $\Omega_{\Lambda+1}$, convergence of $\Omega_{\Lambda+2}$, as well as convergence of IDeC, the locally refined grid $\Omega_{\Lambda+3}$ is introduced, and so on up to and including $\Omega_{L}$.

Before investigating shock-tip and shock-foot flows, we first give an impression of the adaptive method's performance by making a comparison between a nonadaptive and an adaptive grid computation for the standard NACA0012-airfoil flow at $M_{\infty}=0.8, \alpha=1.25^{\circ}$. The coarsest grid used is an $8 \times 5$ O-type grid. In the globally refined grid computation, the finest grid to be considered is the $128 \times 80$ grid $\Omega_{4}$. In the adaptive grid computation, the basic grid $\Omega_{\Lambda}$ is already the $16 \times 10$ grid $\Omega_{1}$. On top of $\Omega_{\Lambda}=\Omega_{1}$, local refinements are introduced up to and including $\Omega_{4}$. A cell is marked to be refined when, in that cell for the density $\rho$, the first undivided difference in flow direction is larger than 0.02 , or when the same difference normal to flow direction is larger than 0.004 . (The choice for the undivided difference of density has been taken over from Dannenhoffer (1991).) In Figure 9 a detail is given of the globally refined grid $\Omega_{4}$, as well as of the corresponding, converged, locally refined, composite grid. The corresponding converged surface pressure distributions are given in Figure 10(a). Notice that they are practically identical. Notice particularly that the upper and lower shock locations, which are very sensitive to, e.g., offenses against the conservation laws (Leveque, 1990), are the same in the uniform and the locally refined grid solutions. The results indicate that the discretization applied at the fine-coarse grid boundaries is second-order accurate and conservative. To show the solution method's convergence behavior, in Figure 10(b) the logarithm of the mean of the four $L_{1}$-norms of the residual is given versus the total number of Newton iteration steps over all cells. Numerical experiments (Van der Maarel, 1993) indicate that, for both the adaptive-grid code 

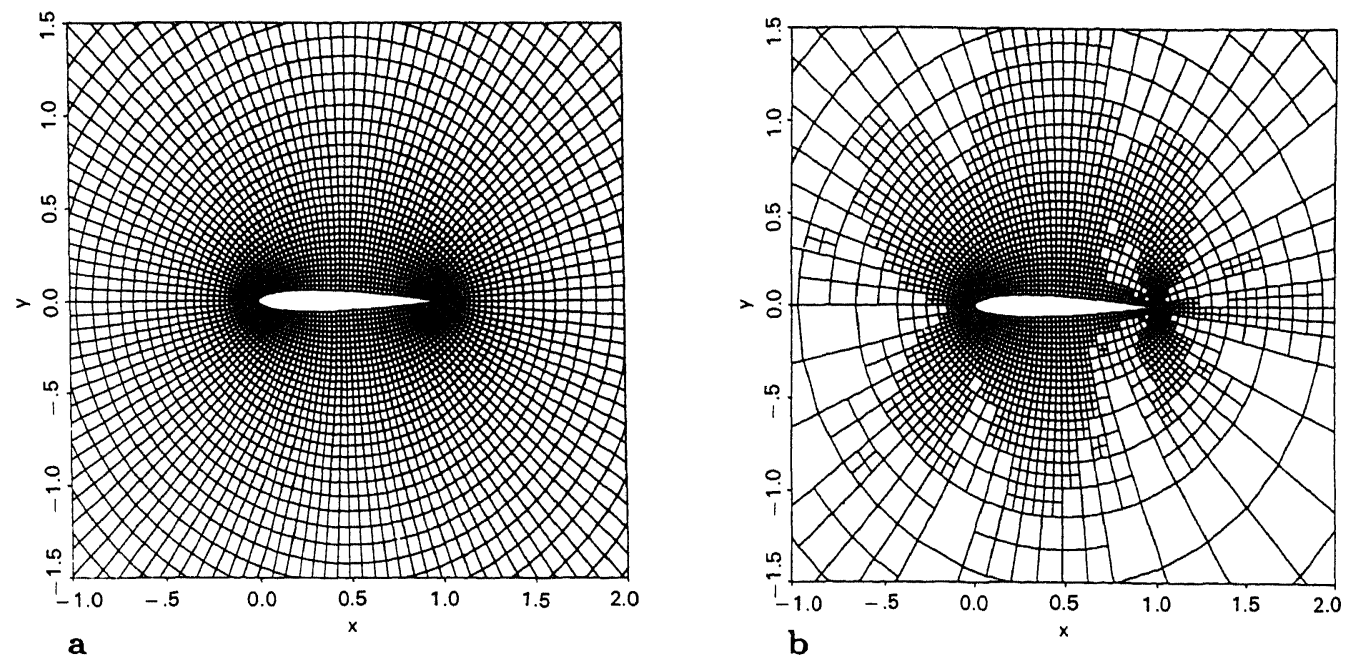

Figure 9. Detail of grid around NACA0012-airfoil, $L=4$ : (a) globally refined and (b) converged, locally refined, composite.

(with its dedicated quad-tree data structure) and the uniform-grid code (with its dedicated direct data structure), the total number of Newton iteration steps performed (over all cells) is a nearly direct measure for the total CPU time spent. Further, the experiments show that in the adaptive-grid codeincluding the overhead of all other numerical actions-a single Newton iteration step is about $33 \%$ more expensive than in the uniform-grid code. Interpreting the convergence results in Figure $10(\mathrm{~b})$ as such, it appears that, to obtain fully converged solutions which have the same accuracy (Figure 10(a)), the locally refined grid algorithm appears to be about four times more efficient than the globally refined grid algorithm (which is already quite efficient itself).

\section{Numerical Experiments}

\subsection{Test Cases}

Numerical computations are performed for flows of a di-atomic, perfect gas $\left(\gamma=\frac{7}{5}\right)$ around a NACA0012-airfoil. The computations are started-in a nonadaptive way-on the same coarsest grid $\Omega_{0}$ as applied for the previous standard test case: a uniform $8 \times 5$ O-type grid. The far-field boundary

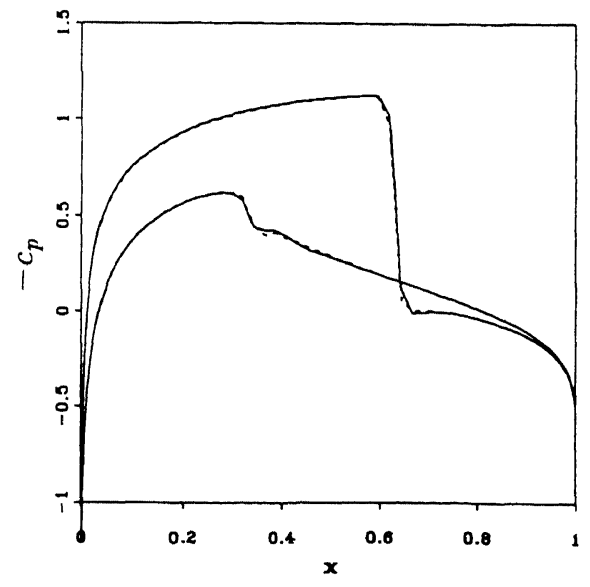

a

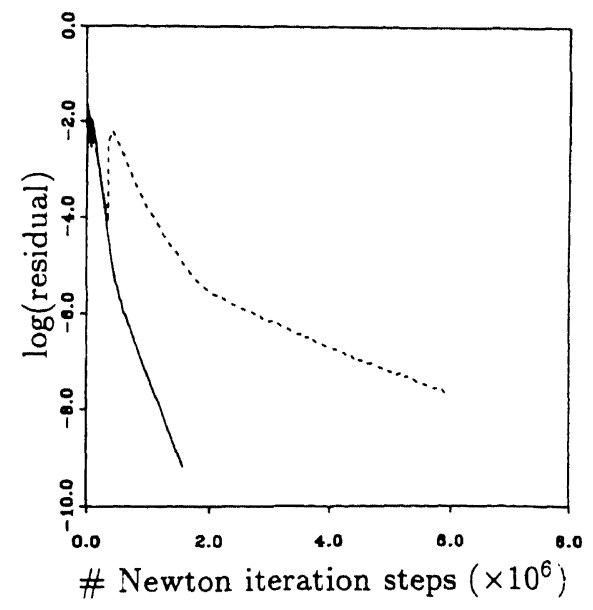

b

Figure 10. Results of NACA0012-airfoil at $M_{\infty}=0.8, \alpha=1.25^{\circ} ;---$ : globally refined, - : locally refined: (a) surface pressure distributions and (b) convergence histories. 
is located at about 100 chord lengths from the airfoil. Here the uniform grid computations are not stopped at $\Omega_{\Lambda}=\Omega_{1}$, but, instead, at $\Omega_{\Lambda}=\Omega_{3}$. On top of $\Omega_{3}$, local refinements are introduced. Details about the refinement procedure are discussed in Section 3.2. Also here, in the nested iteration from $\Omega_{0}$ up to and including $\Omega_{\Lambda}=\Omega_{3}$, the discrete equations solved are first-order accurate only. Starting from $\Omega_{3}$ we begin to solve second-order discretized equations; again those corresponding to the Van Albada scheme.

The following two far-field boundary-condition cases are considered:

- $M_{\infty}=0.8, \alpha=0$.

- $M_{\infty}=0.8, \alpha=\alpha_{M_{1}^{*}}$.

The special incidence $\alpha_{M_{1}^{*}}$ is that at which $M_{1}=M_{1}^{*}$. The first, symmetrical test case is arbitrarily chosen, so far as that it is known to yield a flow with a shock wave for which $M_{1}<M_{1}^{*}$ ( $\approx 1.662$ for $\gamma=\frac{7}{5}$ ). Hence, for the first test case a postshock pressure correction is expected. The shock-tip flow is also studied. The second test case, with $\alpha=\alpha_{M_{1}^{*}}$, is studied to verify whether in this case the numerical shock-foot flow is without any postshock pressure correction and, particularly, whether, in agreement with the solution of Zierep (1958a), it is at the limit of existence. Since the value of $\alpha_{M_{i}^{*}}$ is not known in advance, for the second test case we have to iterate on $\alpha$. This incidence iteration allows the capture of a shock wave on the convex surface, somewhere between nose and tail, and with $M_{1}$ converging to $M_{1}^{*}$ in the limit of the iteration process. (The alternative approach of varying $M_{\infty}$, while keeping $\alpha=0$, cannot lead to $M_{1}=M_{1}^{*}$ for the NACA0012-airfoil.) The incidence iteration is a Newton iteration which is free in the sense that it allows values of $\alpha$ for which $\left(M_{1}\right)_{l}$ lies in the "forbidden" range $\left(M_{1}^{*}, M_{1}^{* *}\right)$. We allow this wittingly in order to investigate the existence of this range.

\subsection{Refinement Procedure}

For the present computations, in which we want to zoom in at the shock tip and shock foot efficiently, a nonstandard refinement criterion is applied. We simply check, for each finite volume on the composite grid, whether, for any of its four cell faces, a sonic point occurs along one of the four corresponding wave paths in state space. If one (or more) sonic points are detected, that cell, as well as some possible buffer zone of neighboring cells on the composite grid, is flagged to be refined. A relevant detail in this wave-path approach is that it is considered in flow direction. In computing the flow direction at each cell face, use is made of the density-dependent relation derived in Koren and Hemker (1992). Though it is also possible to derefine cells which have been refined once but no longer need to be so, here we do not do so. (In applying both refinements and derefinements, for a steady problem, we once obtained periodically oscillating grids.) A doubt that may arise against the appropriateness of the present sonic-pointwise enrichment is that, although the accuracy of the
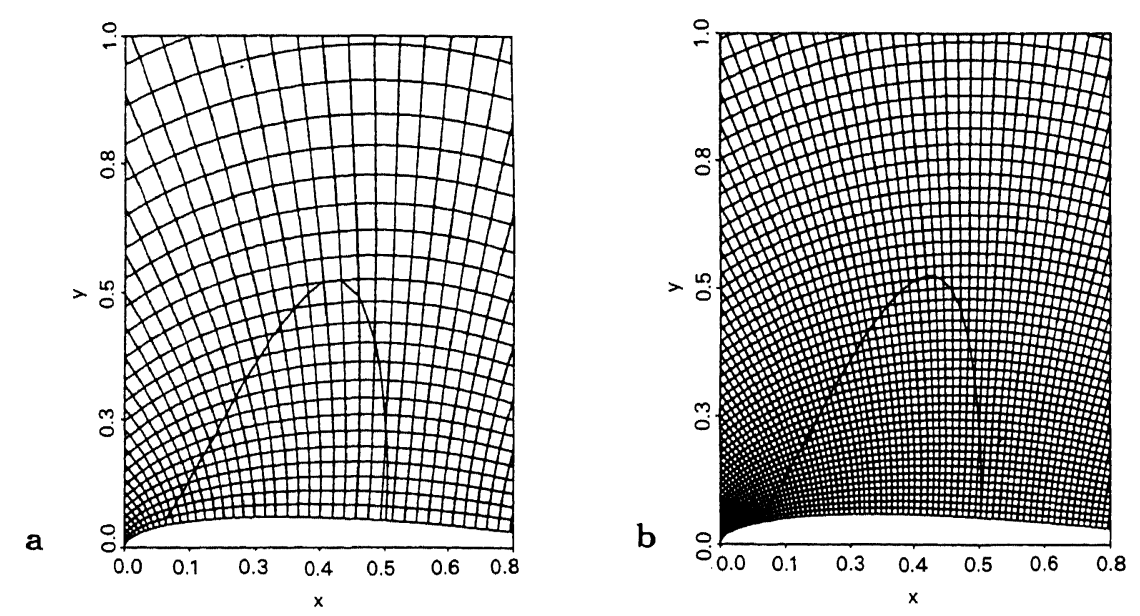

Figure 11. Globally refined grids with sonic lines: (a) $L=4$ and (b) $L=5$. 

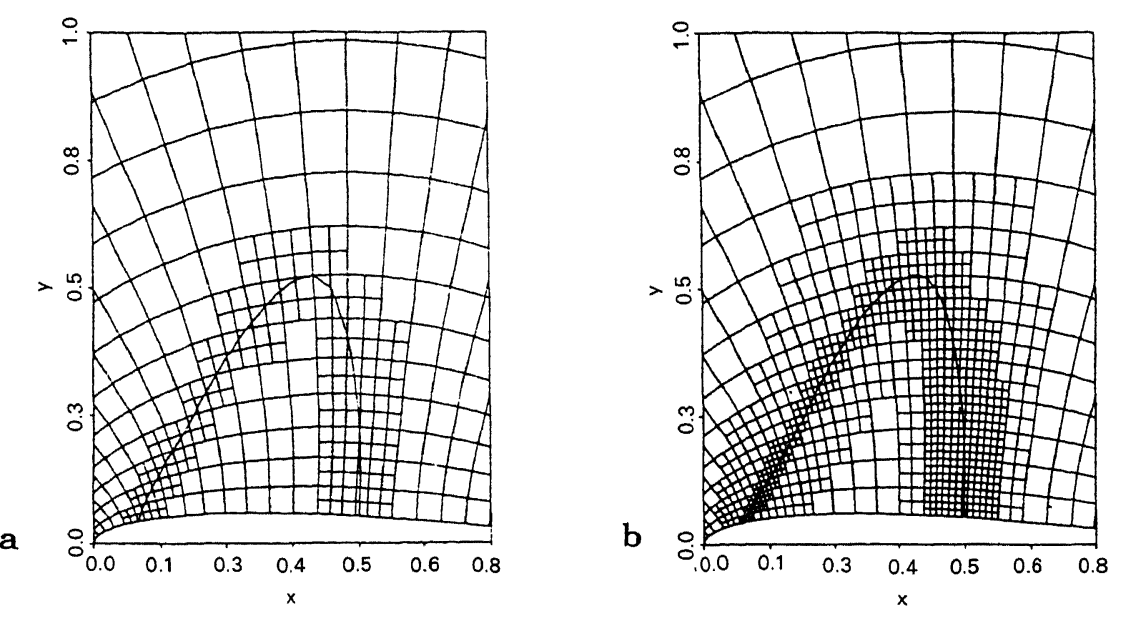

Figure 12. Converged, locally refined, composite grids with sonic lines: (a) $L=4$ and (b) $L=5$.

numerical solution is improved, the degree of improvement may be small, because cells with relatively large errors may remain unrefined and hence persistent in keeping the global solution accuracy low; global flow features may be missed (Warren et al., 1991). We try to counteract this by the choice of a rather fine basic grid $\left(\Omega_{\Lambda}=\Omega_{3}\right)$. To investigate whether the basic grid $\Omega_{\Lambda}$ is fine enough, indeed to prevent the present nonstandard refinement criterion leading to a loss of global flow features, for the first test case to be considered $\left(M_{\infty}=0.8, \alpha=0\right)$ we compare (converged) solutions obtained on the (converged) locally refined, composite grids $\Omega_{4}$ and $\Omega_{5}$ (Figure 12) with the corresponding solutions on the globally refined grids $\Omega_{4}$ and $\Omega_{5}$ (Figure 11). The buffer zone of flagged cells was taken equal to zero. The corresponding sonic lines are depicted on all four grids. Notice that hardly any differences can be observed between sonic lines on the corresponding locally and globally refined grids (Figures 11 and 12). To make a clearer comparison, in Figure 13 surface pressure distributions are given. The agreement between locally and globally refined-grid solutions is good and makes us conclude that the present, nonstandard refinement criterion can be applied safely.

\subsection{Numerical Results}

3.3.1. Results for $\alpha=0$. For $\alpha=0$ we proceed by considering $L=6$ and 7 , keeping the buffer zone of flagged cells equal to zero. In Figure 14 a detail of the converged composite grids is given, again with sonic lines.

In Figure 15 the corresponding upper surface pressure distributions are given. Clearly visible in the latter figures is the occurrence of a postshock expansion. We proceed by investigating the steepness of this expansion for decreasing mesh width. Assuming that in the exact solution, in conflict with theory,
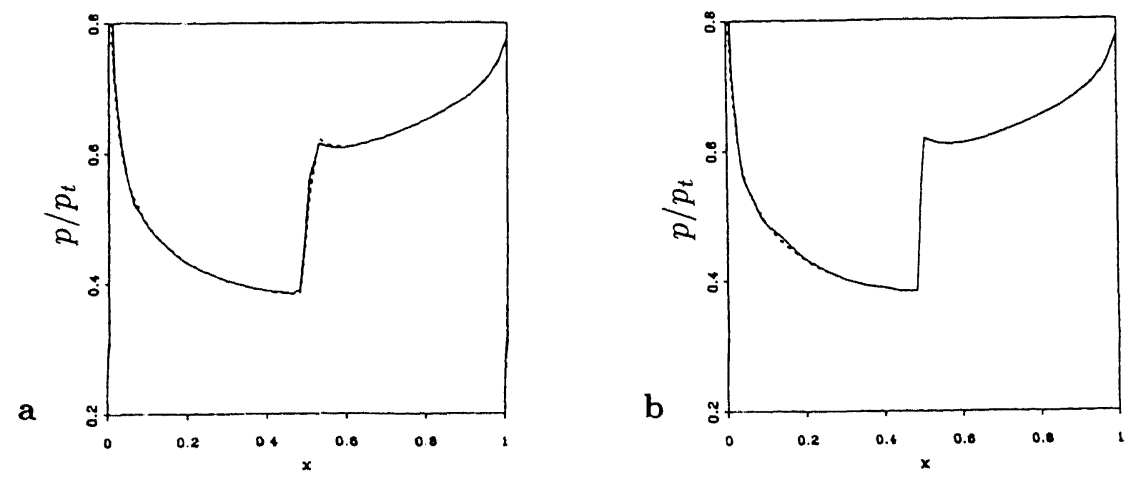

Figure 13. Surface pressure distributions, $\alpha=0 ;---$ : globally refined, - : locally refined; (a) $L=4$ and (b) $L=5$. 


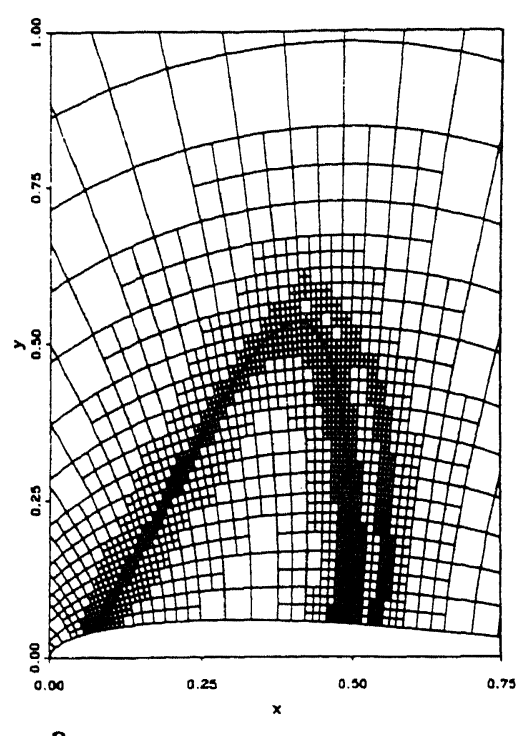

a

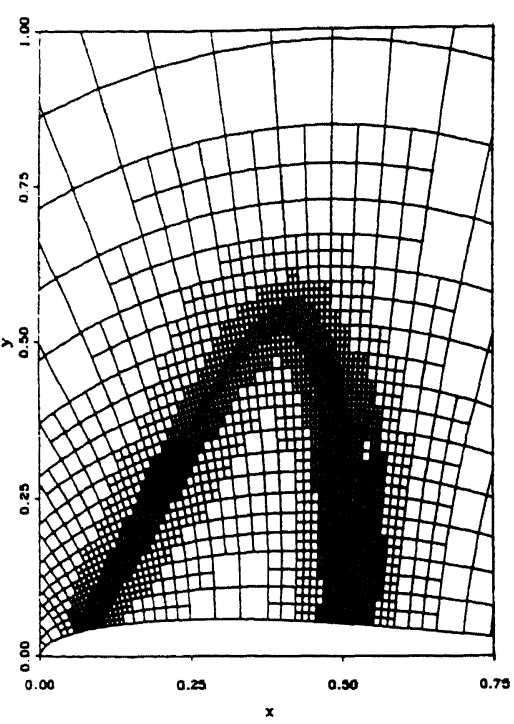

b

Figure 14. Converged composite grids with sonic lines, $\alpha=0$ : (a) $L=6$ and (b) $L=7$.

no infinitely steep postshock expansion is present $\left(\partial p_{2} / \partial x>-\infty\right)$, we introduce the following error $\delta_{L}$ :

$$
\delta_{L} \equiv\left(\frac{\Delta p_{2}}{\Delta x}\right)_{L}-\frac{\partial p_{2}}{\partial x}
$$

which we assume to be positive for all $L$. Then, given the assumed finity of the exact postshock expansion and given the formal second-order accuracy of the discretization method applied, for sufficiently large $L$ we can certainly write

$$
\delta_{L+1}=\left(\frac{1}{2}\right)^{p} \delta_{L}, \quad p>0,
$$

where $p$ denotes the local order of accuracy of the discretization method in the postshock region. Given relations (20a)-(20b) and the previous assumptions, it should hold that

$$
\left(\frac{\Delta p_{2}}{\Delta x}\right)_{L-1}-\left(\frac{\Delta p_{2}}{\Delta x}\right)_{L}>\left(\frac{\Delta p_{2}}{\Delta x}\right)_{L}-\left(\frac{\Delta p_{2}}{\Delta x}\right)_{L+1}
$$

In Table 1 the values of $\left(\Delta p_{2} / \Delta x\right)_{L}$ are given that are actually found in the numerical experiments for $L=5,6$, and 7. It appears that relation (20c) is not satisfied by the numerical results. Hence, one of

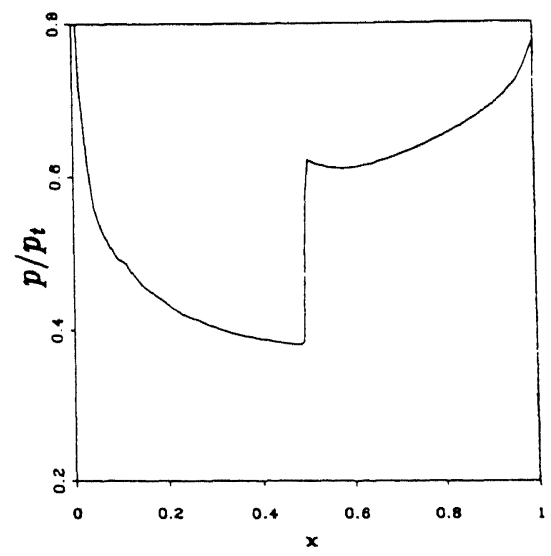

a

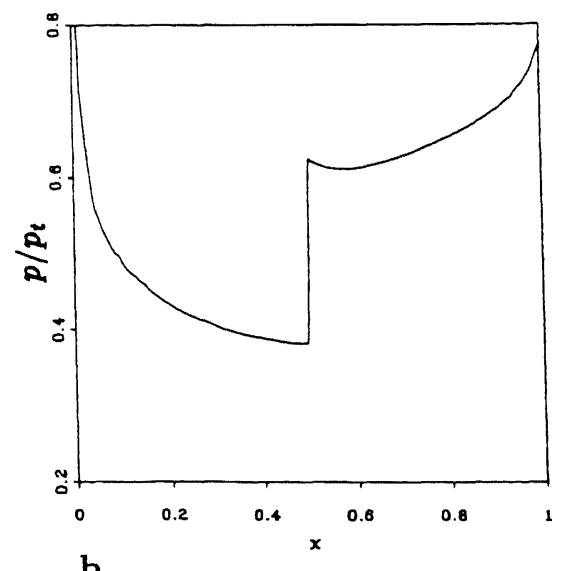

b

Figure 15. Surface pressure distributions, $\alpha=0$ : (a) $L=6$ and (b) $L=7$. 
Table 1. Postshock expansions, $\alpha=0$.

\begin{tabular}{cccc}
\hline & \multicolumn{3}{c}{$L$} \\
\cline { 2 - 4 } & 5 & 6 & 7 \\
\hline$\left(\frac{\Delta p_{2}}{\Delta x}\right)_{L}$ & -0.28 & -0.36 & -0.50 \\
\hline
\end{tabular}

our assumptions (regular solution, sufficiently large $L$ ) is not satisfied. Assuming that $L=5,6$, and 7 is sufficiently large-in agreement with theory-the results show that the numerical postshock expansion converges to an infinitely steep expansion.

Concerning the shock-tip flow, for all values of $L$ considered, we do not observe the triple-shock configuration as advocated by Kraiko (1985). The configurations found are comparable with the one which is supported by Germain and Gillon (1961); in all cases the shock does not bifurcate (see Figure 16 for one detailed view of this). The subtle inflection point is not (yet) observed.

3.3.2. Results for $\alpha=\alpha_{M_{1}^{*}}$. For $\alpha=\alpha_{M_{1}^{*}}$ we have results for $L=4$ and 5 only, with the buffer zone-for reasons of robustness - taken two cells thick. Analogous to the previous results, in Figure 17 we give the converged composite grids with corresponding sonic lines and Figure 18 gives the corresponding surface pressure distributions. Notice that the postshock expansion has indeed vanished. In agreement with theory, downstream of the shock wave, the two surface pressure distributions in Figure 18 nicely show a pressure increase only. A remarkable phenomenon that we observe here during the incidence iteration is that $\mathrm{IDeC}$ does not converge (but also does not diverge) as long as $\left(M_{1}\right)_{L}$ is in the range $\left(M_{1}^{*}, M_{1}^{* *}\right)$. The reason why we show results for $L=5$ at a maximum only, is that IDeC diverges for $L \geq 6$. (With a buffer zone of less than two cells, IDeC even diverges for $L=5$.) Concerning the shock-tip flow, similar to the results for $\alpha=0$, the configurations found agree best with those of Germain and Gillon (1961).

The convergence problems that arise for this test case seem to indicate that, indeed, no steady shock wave perpendicular to the wall can exist in some range with $M_{1}^{*}$ as the lower bound. A question which then arises is: if there is a steady flow solution beyond $M_{1}^{*}$ which is still tangent to the wall, what does that flow solution look like? A conjecture that we have is that a steady normal shock wave at $M_{1}^{*}$ (a normal shock with normal extension) may bifurcate (with increasing $M_{1}$ ) into two steady oblique shock waves (Figure 19(a)): a major oblique shock as the remainder of the single shock at $M_{1}^{*}$ and-in front of that-a minor oblique shock, which has just developed. (Both shocks have a common foot and stand oblique on the wall in such a way that the flow remains tangent to the wall when passing through the double-shock foot.) Starting from a configuration as depicted in Figure

Figure 16. Mach number distribution around shock tip, $L=$

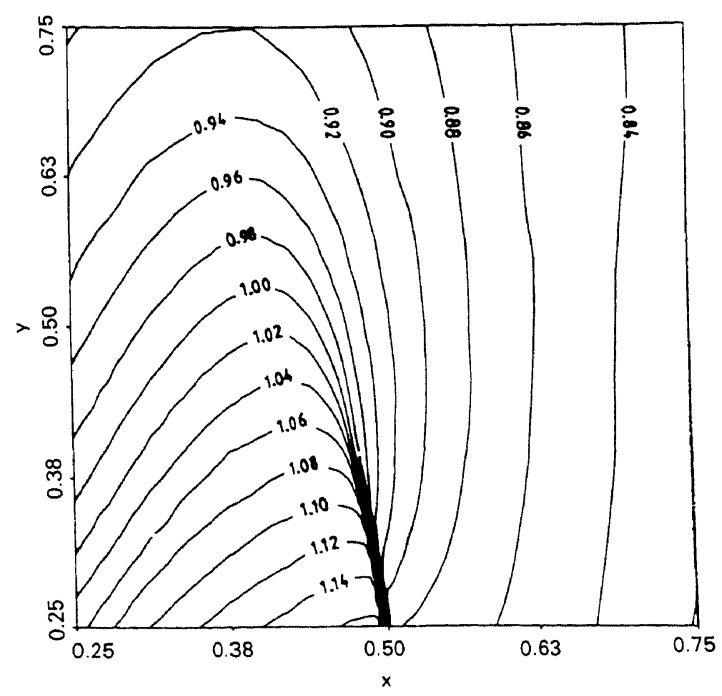




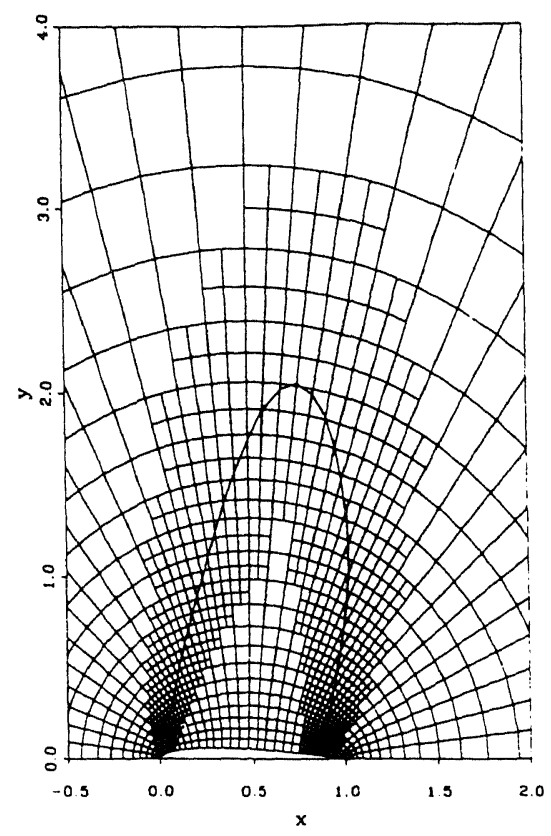

a

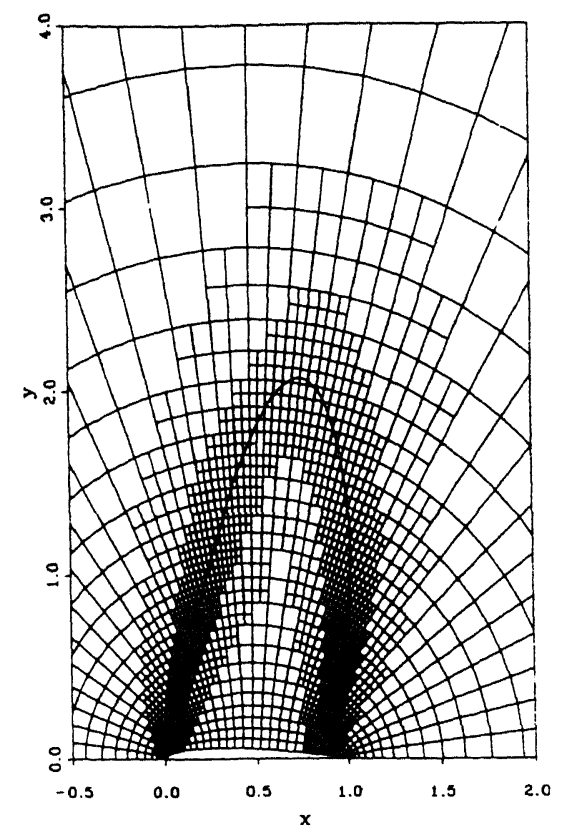

b

Figure 17. Converged composite grids with sonic lines, $\alpha=\alpha_{M_{i}^{*}}$ (a) $L=4$ and (b) $L=5$.

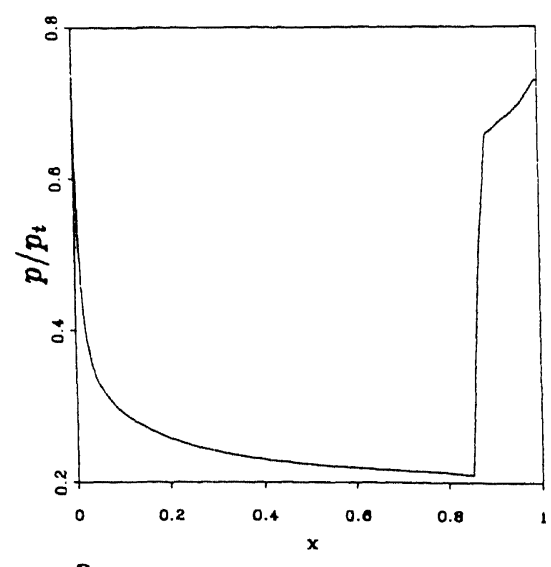

a

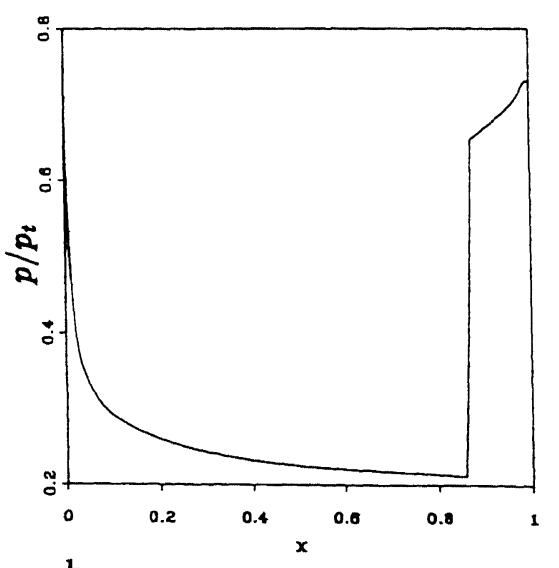

b

Figure 18. Surface pressure distributions, $\alpha=\alpha_{M_{1}^{*}}$ : (a) $L=4$ and (b) $L=5$.
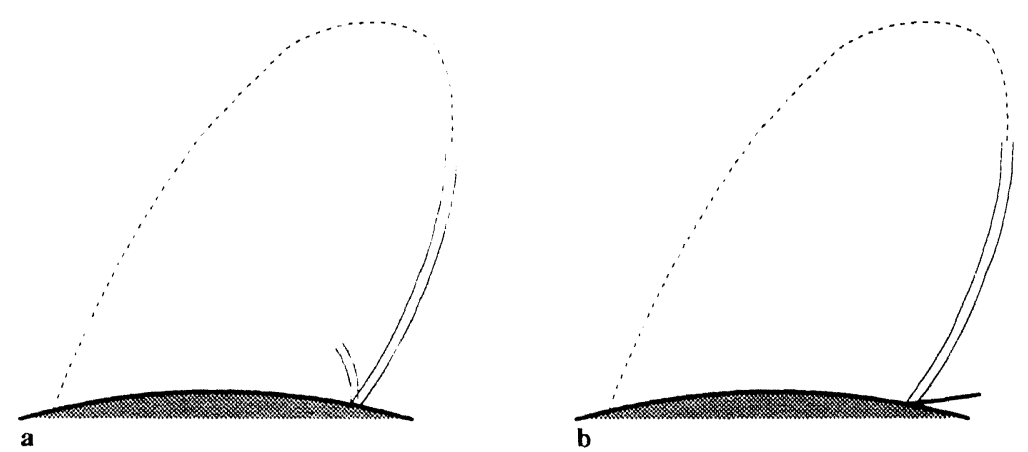

Figure 19. Possible oblique shock-foot configurations beyond normal, single-shock-foot configuration at $M_{1}^{*}$ : (a) double-shock foot (without separation) and (b) single-shock foot (with separation). 
19(a), for decreasing Mach number, the acute angle between both oblique shock waves in their common foot is supposed to converge to zero; the double-shock-foot configuration is supposed to transform into the normal, single-shock-foot configuration at $M_{1}^{*}$ (vice versa, for increasing Mach number, the minor shock is supposed to increase in strength, size, and obliquity.) A nice feature of the hypothetical double-shock-foot configuration in Figure 19(a) is its possible relation with he tripleshock-tip configuration from Figure 7(b). Whereas the single-shock-tip configuration from Figure 7(a) could be considered as a special case of this triple-shock-tip configuration, namely, with the shocktriple-point in the highest possible position, the double-shock-foot configuration from Figure 19(a) may be considered as the other extreme case of the shock-triple-point configuration, namely, with the shock-triple-point in the lowest possible position. From this viewpoint, it might be possible to match analytical solutions for the flow in the shock-tip region to those for the flow in the shock-foot region.

Probably, a steady double-shock-foot configuration as depicted in Figure 19(a) can only exist for very specific boundary conditions. Solutions that will certainly prevail over this double-shock-foot solution are those which simply have an oblique single-shock foot (Figure 19(b)). In these flow solutions the flow is separated from the wall in passing through the shock foot and no unique (inviscid) solution exists in the separation zone. These solutions are probably mostly unsteady.

Concerning this supposed unsteadiness, interesting results can be found in Dervieux et al. (1989), where, for a circular cylinder at $M_{\infty}=0.5$, Euler flow results are presented which have been obtained by various numerical methods. It is interesting to see that when using unsteady numerical methods for this circular-cylinder test-case, fully subsonic initial solutions first seem to evolve to a specific quasi-steady, transonic solution, which becomes unsteady when continuing the time integration. Inspecting all circular-cylinder results in Dervieux et al. (1989), our conjecture is that the aforementioned, specific quasi-steady solution is at $M_{1}=M_{1}^{*}$; it seems that the genesis of unsteadiness is when $M_{1}$ exactly equals $M_{1}^{*}$. Results which make this particularly plausible are those of Pandolfi and Larocca (1989) and Satofuka and Morinishi (1989).

\section{Conclusions}

In the first part of this paper a critical review was given of known analytical and (as far as existing) numerical results on shock-foot and shock-tip flows. For the shock-foot flow, for which only analytical work exists, a critical review was given of the work which is probably best known for it: that of Zierep (1958a, 1958b). Despite initial doubts about the correctness of Zierep's analytical solution for the shock-foot flow, from our subsequent analytical results no evidence arose that Zierep's solution is wrong in the practically relevant $M_{1}$-range $\left(1, M_{1}^{*}\right), M_{1}^{*} \approx 1.662$ for $\gamma=\frac{7}{5}$. In particular, for the singular shock-foot curvature as found by Zierep for $M_{1}<M_{1}^{*}$ and $M_{1}>M_{1}^{* *}, M_{1}^{* *}=2$ for $\gamma=\frac{7}{5}$, we investigated whether there is a discrepancy between this result and the uncurved-shock relations that Zierep applied in deriving it. We found that in fact only in the limit $M_{1} \downarrow M_{1}^{* *}$ does such a discrepancy exist. Further, we still only disagree with Zierep about the detail that at $M_{1}=M_{1}^{*}$ the shock wave is uncurved in its foot; in our opinion this curvature at $M_{1}^{*}$ is (still) undetermined. A remaining, general criticism on the analytical work of Zierep is that although it was done with the Euler equations (not with, e.g., the TSP equation), it has limited validity because it is local. Numerical fine-grid investigations of the shock-foot flow as part of a much more global flow were (and still are) filling a need. For the shock-tip flow, besides analytical work, numerical work also exists; however, both for the TSP equation only. Moreover, here the analyses are also local only, and the numerics is limited in accuracy due to insufficient grid fineness. Also for the shock-tip flow, fine-grid Euler flow computations were (and still are) filling a need.

The second, numerical part of the paper was motivated by the facts that unanswered questions still exist for both flows, and that, for answers that have been given, models and methods have been used for which the validity is questionable. Our numerical findings are the following. Concerning the shock-foot flow, the numerical results agree with Zierep's analytical results (singular postshock expansion for $M_{1}<M_{1}^{*}$, no postshock expansion at $M_{1}^{*}$, and probably no steady solution for $M_{1}>M_{1}^{*}$ ). Our main suggestion for further research is to make a qualitative study of the different possible shock-foot-flow solutions beyond $M_{1}=M_{1}^{*}$. Given the possible change at $M_{1}=M_{1}^{*}$ from 
steadiness to unsteadiness, such a study might be of practical relevance as well (buffeting). It would be interesting also to invoke the shock-tip configuration in such an analysis. Concerning the shock-tip flow, the present numerical results agree with the analytical results of Germain and Gillon (1961); with the sonic line continuously connected to the shock tip. This may well be the commonly occurring shock-tip configuration.

The solution-adaptive multigrid method applied here has appeared to be accurate and efficient in computing the present shock flow details. It seems to be a good tool for detailed investigations of other singular flow phenomena.

\section{References}

Ackeret, J., Feldmann, F., and Rott, N. (1946). Untersuchungen an Verdichtungsstössen und Grenzschichten in Schnell Bewegten Gasen. Mitteilungen aus dem Institut für Aerodynamik 10.

Berger, M.J., and Jameson, A. (1985). Automatic adaptive grid refinement for the Euler equations. AIAA J., 23, 561-568.

Berger, M.J., and Oliger, J. (1984). Adaptive mesh refinement for hyperbolic partial differential equations. J. Comput. Phys., 53, $484-512$.

Courant. R., and Friedrichs, K.O. (1976). Supersonic Flow and Shock Waves. Springer-Verlag, New York.

Dannenhoffer, III, J.F. (1991). A comparison of adaptive-grid redistribution and embedding for steady transonic flows. Internat. J. Numer. Methods Fluids, 32, 653-663.

Dervieux, A., Van Leer, B., Périaux, J., and Rizzi, A., eds. (1989). Numerical Simulation of Compressible Euler Flows. Vieweg, Braunschweig.

Désidéri, J.-A., and Hemker, P.W. (1990). Analysis of the Convergence of Iterative Implicit and Defect-Correction Algorithms for Hyperbolic Problems. CWI Report NM-R9004.

De Zeeuw, D., and Powell, K.G. (1991). An Adaptively-Refined Cartesian Mesh Solver for the Euler Equations. AIAA Paper $91-1542$.

Fuchs, L. (1990). Calculation of flow fields using overlapping grids. In Proceedings of the Eighth GAMM Conference on Numerical Methods in Fluid Mechanics (P. Wesseling, ed.), pp. 138-147. Vieweg, Braunschweig.

Germain, P., and Gillon, G. (1961). Ecoulements Transsoniques au Voisinage d'un Point de Rencontre d'une Onde de Choc et d'une Ligne Sonique. ONERA Publication 102.

Hafez, M.M., and Cheng, H.K. (1975). Convergence Acceleration and Shock Fitting for Transonic Aerodynamics Computations. AIAA Paper 75-51.

Hemker, P.W., and Spekreijse, S.P. (1986). Multiple grid and Osher's scheme for the efficient solution of the steady Euler equations. Appl. Numer. Math., 2, 475-493.

Hemker, P.W., Van der Maarel, H.T.M., and Everaars, C.T.H. (1990). BASIS: A Data Structure for Adaptive Multigrid Computations. CWI Report NM-R9014.

Koren, B. (1988). Defect correction and multigrid for an efficient and accurate computation of airfoil flows. J. Comput. Phys., 77, 183-206.

Koren, B., and Hemker, P.W. (1992). Multi-D upwinding and multigridding for steady Euler flow computations. In Proceedings of the Ninth GAMM Conference on Numerical Methods in Fluid Mechanics (J.B. Vos, A. Rizzi, and I.L. Ryhming, eds.), pp. 89-98. Vieweg, Braunschweig.

Kraiko, A.N. (1985). On the configuration of shock waves enclosing a local supersonic zone. P.M.M. USSR, 49, 179-183.

Leveque, R.J. (1990). Numerical Methods for Conservation Laws. Birkhäuser, Basel.

Lin, C.C., and Rubinov, S.I. (1948). On the flow behind curved shocks. J. Math. Phys., 27, 105-129.

Lioen, W.M., and Louter-Nool, M. (to appear). EUVEL: An Euler Vector Extension Library. CWI Report.

Murman, E.M. (1974). Analysis of embedded shock waves calculated by relaxation methods. AIAA J., 12, 626-633.

Osher, S., and Solomon, F. (1982). Upwind difference schemes for hyperbolic systems of conservation laws. Math. Comp., 38 , 339-374.

Pandolfi, M., and Larocca, F. (1989). A contribution to the numerical prediction of transonic flows. In Numerical Simulation of Compressible Euler Flows (A. Dervieux, B. Van Leer, J. Périaux, and A. Rizzi, eds.), pp. 292-308. Vieweg, Braunschweig.

Satofuka, N., and Morinishi, K. (1989). Solution of compressible Euler flows using rational Runge-Kutta time stepping scheme. In Numerical Simulation of Compressible Euler Flows (A. Dervieux, B. Van Leer, J. Périaux, and A. Rizzi, eds.), pp. 309330. Vieweg, Braunschweig.

Tsien, H.S. (1947). Flow conditions near the intersection of a shock wave with solid wall boundary. J. Math. Phys., 26, 69-75.

Van Albada, G.D., Van Leer, B., and Roberts, W.W. (1982). A comparative study of computational methods in cosmic gasdynamics. Astronom. and Astrophys., 108, 76-84.

Van der Maarel, H.T.M. (1992). Adaptive multigrid for the steady Euler equations. Comm. Appl. Numer. Methods, 8, 749-760.

Van der Maarel, H.T.M. (1993). A local grid refinement method for the Euler equations. Doctoral thesis, University of Amsterdam.

Van der Maarel, H.T.M., and Koren, B. (1991). Spurious, zeroth-order entropy generation along a kinked wall. Internat. J. Numer. Methods Fluids, 13, 1113-1129. 
Warren, G.P. (1992). Application of multigrid and adaptive grid embedding to the two-dimensional flux-split Euler equations. Comm. Appl. Numer. Methods, 8, 771-784.

Warren, G.P., Anderson, W.K., Thomas, J.L., and Krist, S.L. (1991). Grid Convergence for Adaptive Methods. AIAA Paper 91-1592.

Yu, N.J., and Seebass, A.R. (1976). Inviscid transonic flow computations with shock fitting. In Symposium Transsonicum II (K. Oswatitsch and D. Rues, eds.), pp. 449-456. Springer-Verlag, Berlin.

Zierep, J. (1958a). Der senkrechte Verdichtungsstoss am gekrümmten Profil. Z. Angew. Math. Phys., IXb, 764-776.

Zierep, J. (1958b). Der Senkrechte Verdichtungsstoss am Gekrümmten Profil. DVL Bericht 51. 